\title{
Sterility Test
}

National Cancer Institute

\section{Source}

National Cancer Institute. Sterility Test. NCI Thesaurus. Code C134278.

Tests done under aseptic conditions to ensure that there are no contaminating microorganism present in the sample. 